\title{
Comparison-speed liquid jets
}

\author{
H.H. Shi, H. Sato
}

\begin{abstract}
The performance of a small high-speed liquid jet apparatus is described. Water jets with velocities from 200 to $700 \mathrm{~m} / \mathrm{s}$ were obtained by firing a deformable lead slug from an air rifle into a stainless steel nozzle containing water sealed with a rubber diaphragm. Nozzle devices using the impact extrusion (IE) and cumulation (CU) methods were designed to generate the jets. The effect of the nozzle diameter and the downstream distance on the jet velocity is examined. The injection sequences are visualized using both shadowgraphy and schlieren photography. The difference between the IE and CU methods of jet generation is found.
\end{abstract}

\section{1}

\section{Introduction}

High-speed liquid jets are a typical phenomenon in fluid mechanics. The jets have important industrial applications and academic interest. Therefore, the problem has long been studied worldwide. One can find references from the proceedings of the series of BHR International Conferences on Jetting Technology, the US Water Jet Conference and the Cambridge Conference on Erosion by Liquid and Solid Impact. Recently, Pianthong et al. (2002) and Weeks et al. (2001) listed the applications of jets in rain erosion, cavitation, jet cutting, diesel fuel injection, etc. Momber (2001) and Kennedy and Field (2002) presented new results of abrasive water jet impact force and high-speed liquid impact on natural materials. From an academic point of view, Shi and Takayama (1999) and Shi et al. (2003a) emphasized that a supersonic liquid jet generator can be used as a fundamental tool in aerodynamics studies. Shi and Itoh (1996) reported the design of and

Received: 23 June 2003 / Accepted: 24 August 2003

Published online: 7 October 2003

(C) Springer-Verlag 2003

H.H. Shi $(\bowtie)$

State Key Laboratory of Nonlinear Mechanics,

Institute of Mechanics,

Chinese Academy of Sciences,

15 Beisihuanxi Road, 100080 Beijing, China

E-mail: hhshi@imech.ac.cn

Fax: +86-10-62579511

H. Sato

Department of Mechanical Engineering,

Nagoya Institute of Technology,

Gokiso-cho, 466-8555

Showa-ku, Nagoya, Japan experiment with a small high-speed liquid jet apparatus based on Bowden and Brunton's (1958) method of firing a deformable lead slug from an air rifle into a stainless steel nozzle containing water sealed with a rubber diaphragm. Because the slug directly impacts the nozzle to extrude the liquid flowing through a narrow orifice, the method is often called the impact extrusion (IE) method or direct impact method. It is known that the quality and behavior of the jets generated largely depend on the generation method or the design of the nozzle device. In an experiment with a high-pressure helium gas gun, Shi et al. (1996, 2003b) demonstrated that a greater jet velocity can be obtained using the cumulation (CU) method, by which the liquid cylinder is first accelerated by the impact of a projectile and then the liquid is accelerated further after entering a converging nozzle. This paper describes an investigation by jet velocity measurement and flow visualization into the effect on the jet generation when the $\mathrm{CU}$ method is applied in a small high-speed liquid jet apparatus.

\section{2}

\section{Experimental devices}

A general schematic of the small high-speed liquid jet apparatus is shown in Fig. 1a. The total length of the apparatus is $1.2 \mathrm{~m}$. A UD-II Sharp $\mathrm{CO}_{2}$ rifle with a bore diameter of $5 \mathrm{~mm}$ is fixed to steel blocks. The gun muzzle is coupled with the flight chamber and is facing a nozzle assembly. Figure $1 \mathrm{~b}$ is a photograph of the apparatus.

Figure 2 shows the nozzle device of the IE method. A lead slug of diameter $5 \mathrm{~mm}$, length $6.7 \mathrm{~mm}$, and mass $0.75 \mathrm{~g}$ directly impacts a stainless steel nozzle. The test nozzles contain about $150 \mathrm{~mm}^{3}$ water and have $0.5 \mathrm{~mm}$, $1 \mathrm{~mm}$, and $2 \mathrm{~mm}$ diameters respectively. The back end diameters of the nozzles are all $5.5 \mathrm{~mm}$, and the liquid is sealed with a 1-mm-thick rubber diaphragm. The slug impacts the diaphragm and pushes the liquid to flow through the nozzle exit. The arrangement for fixing the nozzle in the nozzle holder is shown in the figure. The nozzle is adjacent to a spacer ring and is pressed by a strangleholder screw. The spacer ring can adjust the distance between the nozzle and the screw. Figure 3 shows the nozzle device in the CU method. Now the nozzle is adjacent to a stainless steel cylinder in which $150 \mathrm{~mm}^{3}$ water is sealed between a 9- $\mu$ m-thick Mylar film and a 1 -mm-thick rubber diaphragm. The nozzle and the cylinder are fixed in the nozzle holder by the stranglehold screw. The slug impacts on the diaphragm and push the liquid cylinder to enter the nozzle. After traveling through 

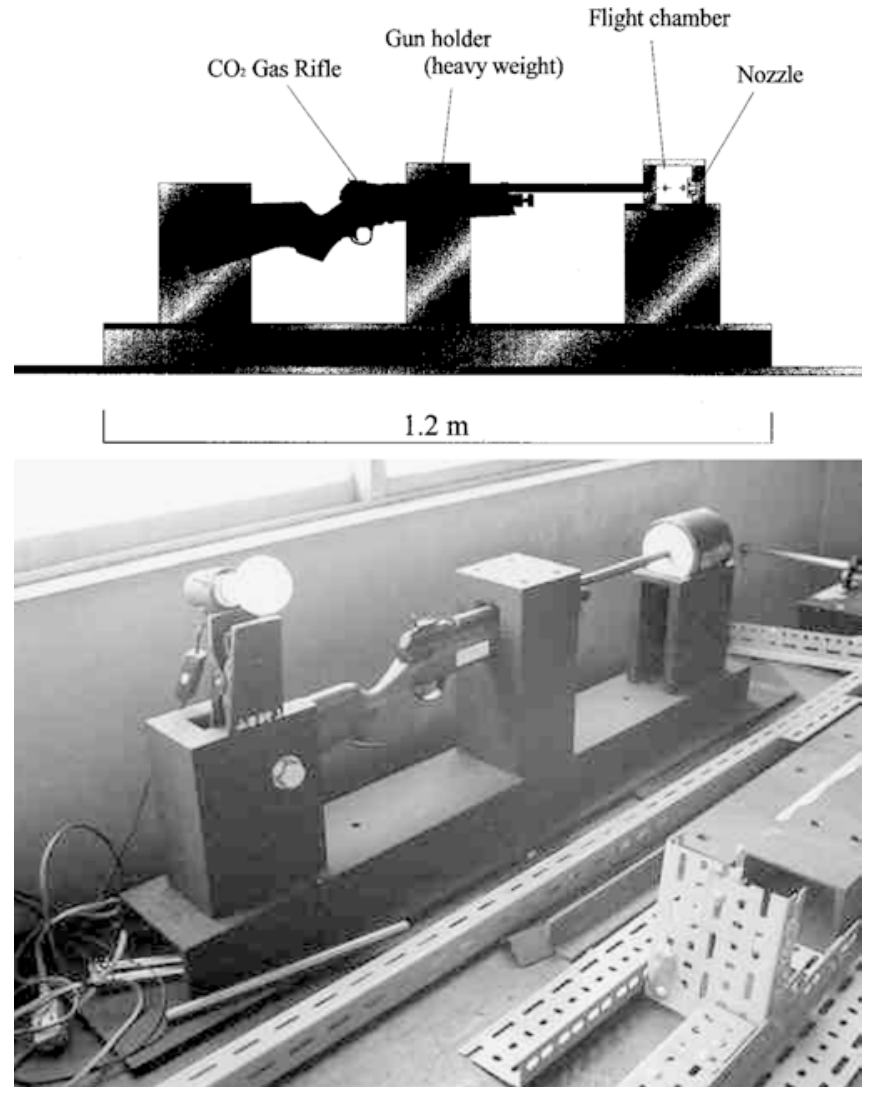

Fig. 1. a Schematic of the small high-speed liquid jet apparatus. b Photograph of the small high-speed liquid jet apparatus

the nozzle, the liquid flows out of the nozzle exit to form a jet. The design techniques of the IE and CU methods are that, before the experiment, (1) for the IE method, the nozzle is fully filled with water (Fig. 2); (2) for the CU method, water only occupies the back section of the nozzle, whereas the front section (including the converging part) of the nozzle is empty (Fig. 3).

Figure 4 shows the system for measuring the velocities of the slug and the water jet. Two laser beams are set inside the flight chamber to measure the slug velocity. The two laser beams are separated by $40 \mathrm{~mm}$, and the first one is $20 \mathrm{~mm}$ from the muzzle exit. The other two laser beams are set at the nozzle exit. The two beams are separated by $15 \mathrm{~mm}$, and the distance between the first one to the nozzle exit $X$ is variable. To examine the effect of the

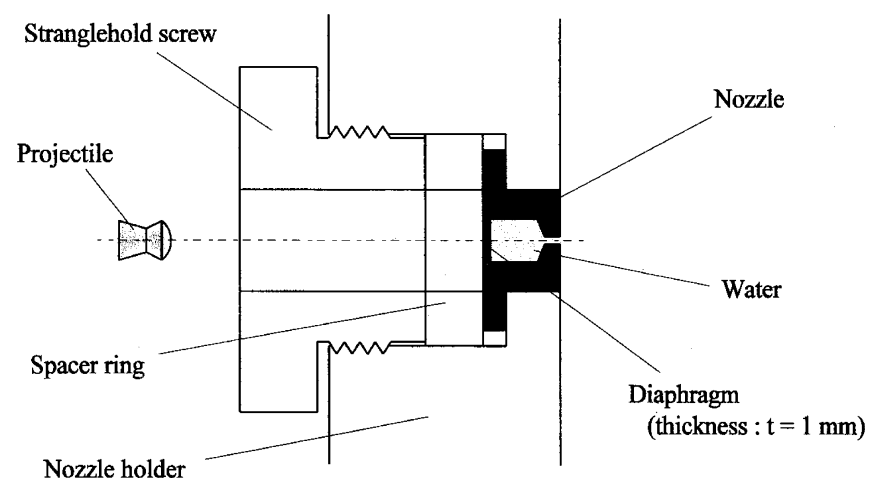

Fig. 2. Nozzle device for the IE method nozzle diameter on the jet velocity, the distance $X$ is kept at $3.5 \mathrm{~mm}$, whereas to examine the jet velocity along the downstream, it is changed from $3.5 \mathrm{~mm}$ to $163.5 \mathrm{~mm}$ with every $40-\mathrm{mm}$ step. All the laser beams are from semiconductor lasers (wavelength $670 \mathrm{~nm}$, power output $5 \mathrm{~mW}$, beam diameter $3.0 \mathrm{~mm}$, and beam diverging angle $6.0 \times 10^{-3} \mathrm{rad}$ ) and the signals from the photodiodes (PIN type, illumination size $\phi 0.8$, shutting frequency $500 \mathrm{MHz}$ ) are collected into a four-channel digital oscilloscope.

The optical systems of the shadowgraphy and schlieren photography are given in Figs. 5 and 6 respectively. The shadowgraphy is performed using a xenon flash (nano pulse light, $180 \mathrm{~ns}$ exposure time) and an open shutter camera in a dark room. A conducting $0.5-\mathrm{mm}$-diameter carbon rod is set in the flight chamber, which is $40 \mathrm{~mm}$ from the muzzle exit. When the carbon rod is broken by an impacting slug, an electric signal is generated to trigger the xenon flash via the delay unit and the power supply. Adjusting the delay time, the jet events at different injection stages can be photographed. The xenon flash is also used in the schlieren photography (Fig. 6). The two concave mirrors are $150 \mathrm{~mm}$ in diameter. The trigger signal of the light source comes from the laser beam, which is set either at the muzzle exit or at the nozzle exit.

\section{3}

\section{Results}

Figures 7 and 8 give the measured relationships between the jet velocity and the impact slug velocity at three nozzle diameters of $0.5,1$, and $2 \mathrm{~mm}$ for the IE and CU generation methods respectively. The measuring point is at a distance of $X=3.5 \mathrm{~mm}$. The general trend is that with increasing slug velocity, the jet velocity is increased since the impact pressure is approximated to be $P=\rho C V_{\text {pro, }}$, where $\rho, C$, and $V_{\text {pro }}$ are the liquid density, the shock wave velocity, and the slug velocity (Shi et al. 1996). The results of the IE method are close to linear relationships, but the results of the $\mathrm{CU}$ method are not. It is obvious from Figure 7 that the smaller the nozzle diameter, the greater the jet velocity. This is because the fluid pressure is higher in a nozzle with a smaller diameter (Shi et al. 1993, 1994). However, Fig. 8 shows that the CU method causes some overlap in the jet velocity, e.g., the jet velocity of 1-mm nozzle is greater than that of $0.5-\mathrm{mm}$ nozzle at the slug velocity of $170 \sim 180 \mathrm{~m} / \mathrm{s}$. This is associated with the fact that it is easy for the complicated acceleration process in the nozzle to cause unsteadiness of the jets (Shi et al. 2003b). A comparison of Figs. 7 and 8, shows that the jet velocities of the $\mathrm{CU}$ method are generally less than those of the IE method. One exception is that the jet velocity of the CU method is greater than that of the IE method at the slug velocity of $185 \mathrm{~m} / \mathrm{s}$ for a 2 -mm-diameter nozzle. These results are somewhat different from those of Shi et al. (1996, 2003b), but it must be noted that in the highpressure helium gas gun experiment, Shi et al. (1996, 2003b) used a composed brass/nylon projectile with $1.16 \mathrm{~g}$ mass and $200 \sim 300 \mathrm{~m} / \mathrm{s}$ impact velocity. Therefore, it is understood that the role of the CU method in the acceleration of a water jet depends on the momentum of the projectile, the diameter of the nozzle, and the volume of water. The results of the effect of the liquid volume on the 


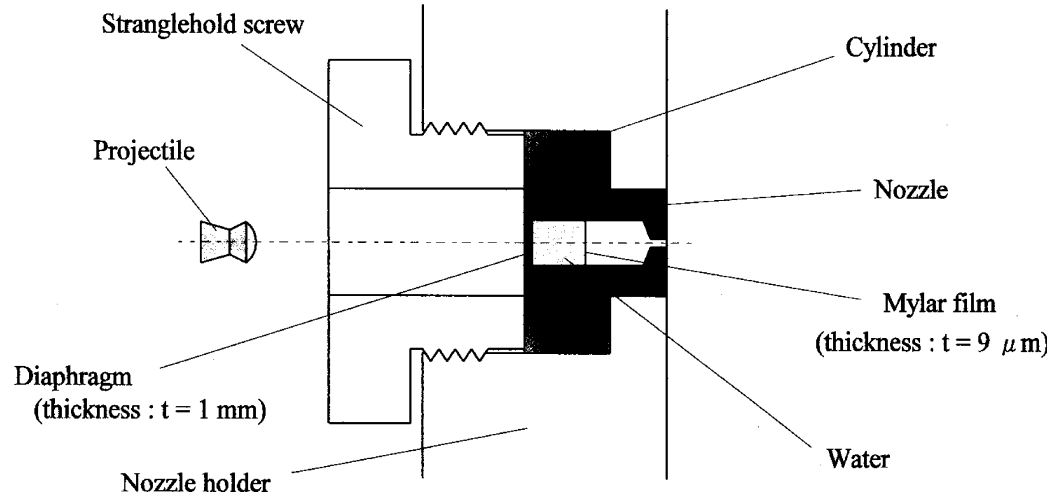

Nozzle holder

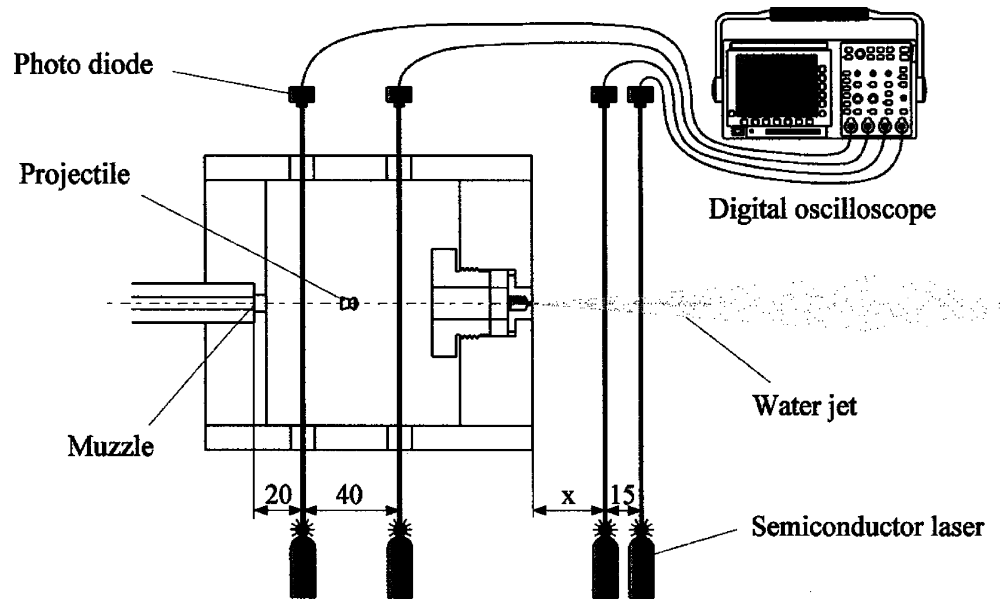

Fig. 3. Nozzle device for the CU method
Fig. 4. Optical system for measuring the jet and slug velocities

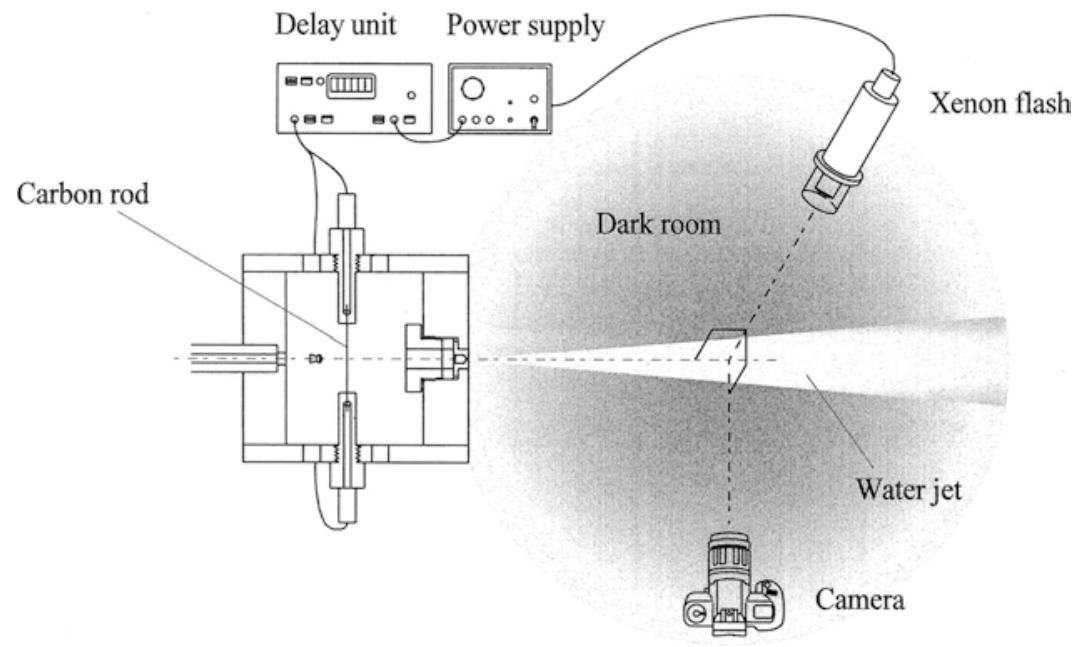

Fig. 5. Optical system of the shadowgraphy for the jets jet generation can be seen in the study of Shi et al. (1993, 2003b).

Figures 9 and 10 give the measured relationships between the jet velocity and the downstream distance for the IE and CU methods respectively. During the experiment and the schlieren photography, the slug velocities are kept around $185 \mathrm{~m} / \mathrm{s}$. For the IE method, the jet velocities of 1- and 2-mm diameter nozzles are relatively stable along the downstream direction. The jet velocity of the $0.5-\mathrm{mm}$ diameter nozzle decreases greatly along the downstream direction, that is, it becomes half the original value at the stand-off distanceof $120 \mathrm{~mm}$. This is because the $0.5-\mathrm{mm}$ diameter jet is severely atomized after leaving the nozzle exit. For the CU method, only the jet velocity of $2-\mathrm{mm}$ diameter nozzle remains stable along the downstream direction. The velocities of the jets from $0.5-$ and $1-\mathrm{mm}$ diameter nozzles are decreased significantly with increase in the stand-off distance.

Figures 11 and 12 give the shadowgraphs of supersonic water jets from the 1-mm-diameter nozzle at different stand-off distance for the IE and CU methods respectively. The arrows in the photos mark the position of the nozzle 


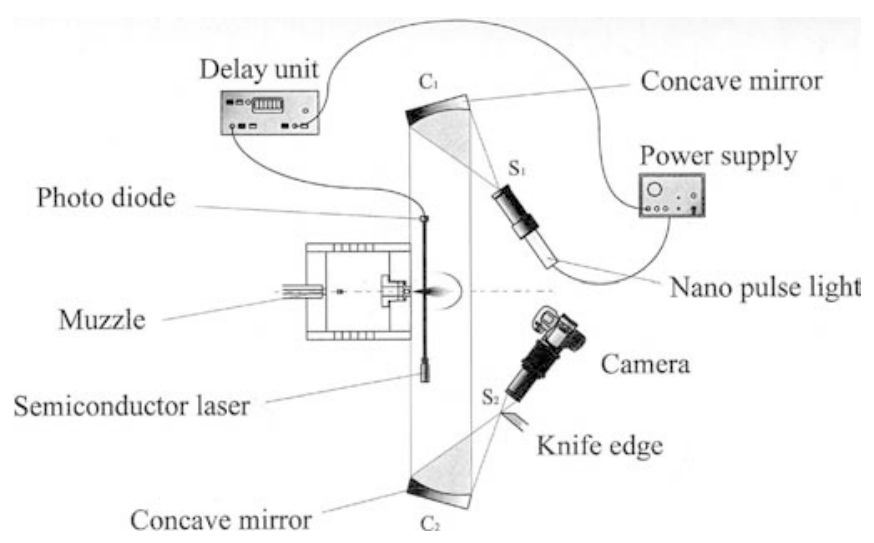

Fig. 6. Optical system of the schlieren photography for the jets

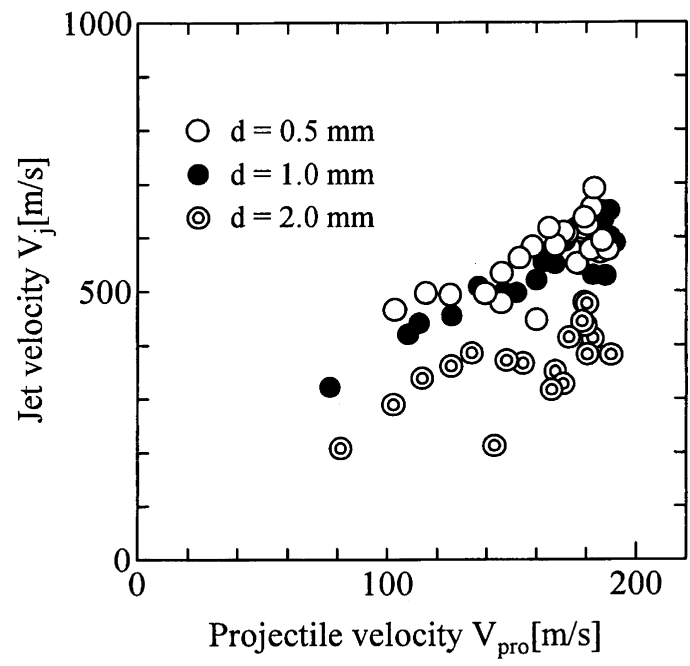

Fig. 7. Relationships between the jet velocity and the slug velocity. IE method. $X=3.5 \mathrm{~mm}$

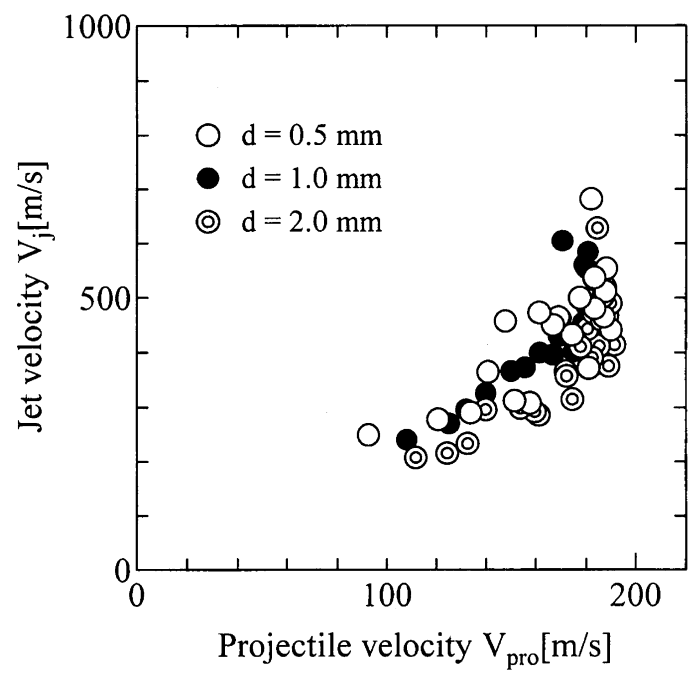

Fig. 8. Relationships between the jet velocity and the slug velocity. CU method. $X=3.5 \mathrm{~mm}$

exit. Figures 13 and 14 give the schlieren photographs of the supersonic water jets from the 1-mm-diameter nozzle at different stand-off distance for the IE and CU methods

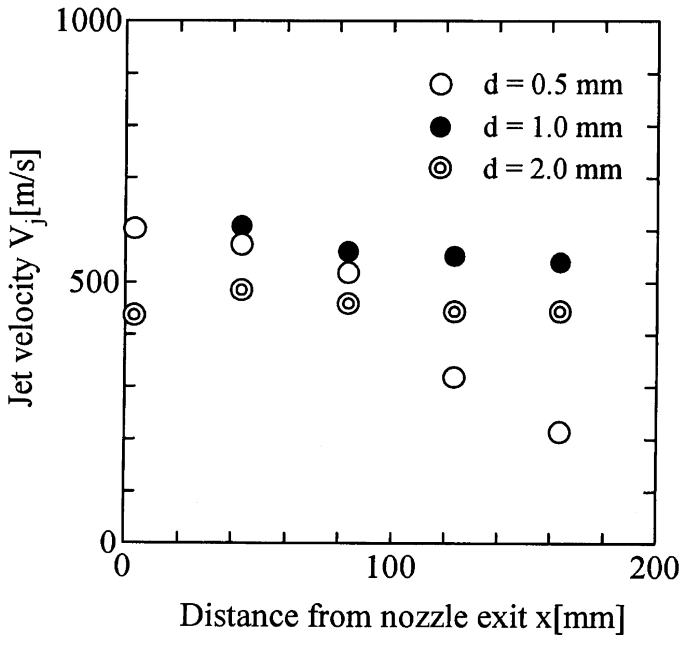

Fig. 9. Relationships between the jet velocity and the stand-off distance. IE method

respectively. We first analyze the jets for the IE method shown in Figs. 11 and 13. In accordance with the jet shape and its stand-off distance, the jets shown in Fig. 11a, b have about a $15 \mathrm{~mm}$ stand-off distance and correspond to the cases shown in Fig. 13e-g. The jets in Fig. 11c-d correspond to the cases in Fig. 13i-j. It is shown that, when the jet is fully developed (Figs. 11c-d and $13 \mathrm{i}-\mathrm{j}$ ), the jet has a sharp supersonic tip and a long stretched thin body which is surrounded by the bifurcated jets near the nozzle exit. The combined shadowgraphy and schlieren photography have revealed the mechanism of the formation of the bifurcated jets. Figures $11 \mathrm{a}-\mathrm{b}$ and $13 \mathrm{a}-\mathrm{h}$ show that, at the beginning, a thin jet of about $1 \mathrm{~mm}$ diameter appears at the nozzle exit (Fig. 13a). Due to the multiple reflection of the shock wave in the nozzle (Shi and Itoh 1999), the successive liquid must flow out of the nozzle (Fig. 13b-c). The successive liquid impacts on the front jet head to cause the liquid to expand radially. As shown in Fig. 11a-b, the diameter of the spray head was $13.3 \mathrm{~mm}$. This is the so-called liquid jet discontinuity (Dunne and Cassen 1956). The radial expansion brings about velocity

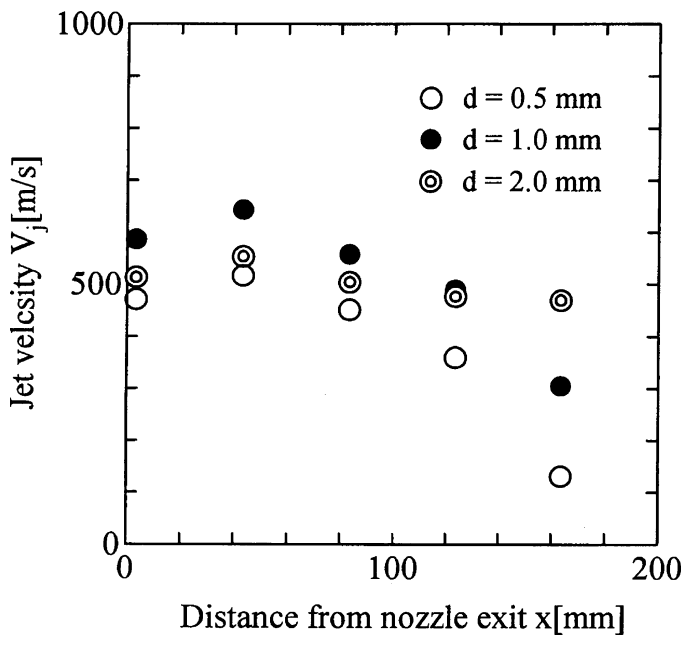

Fig. 10. Relationships between the jet velocity and the stand-off distance. CU method 


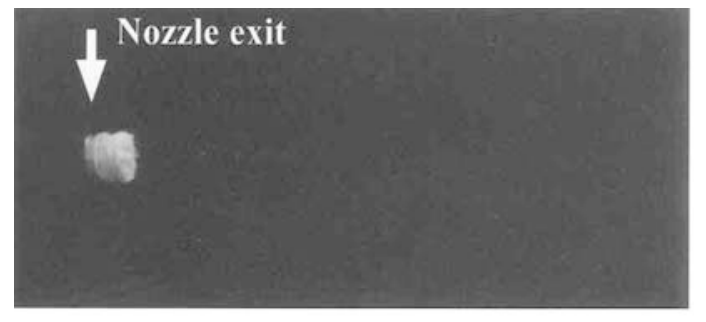

(a) Delay time $380 \mu \mathrm{s}, \mathrm{V}_{\text {pro }}=186 \mathrm{~m} / \mathrm{s}$

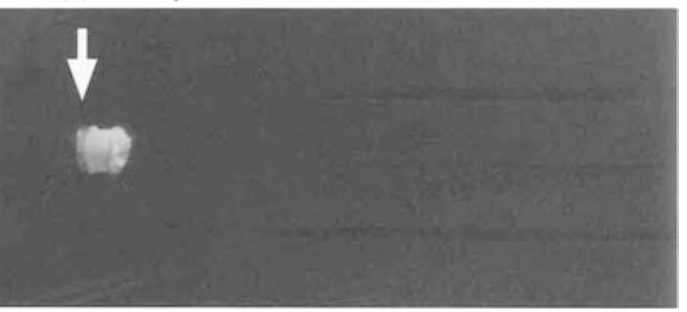

(b) Delay time $400 \mu \mathrm{s}, \mathrm{V}_{\mathrm{pro}}=179 \mathrm{~m} / \mathrm{s}$

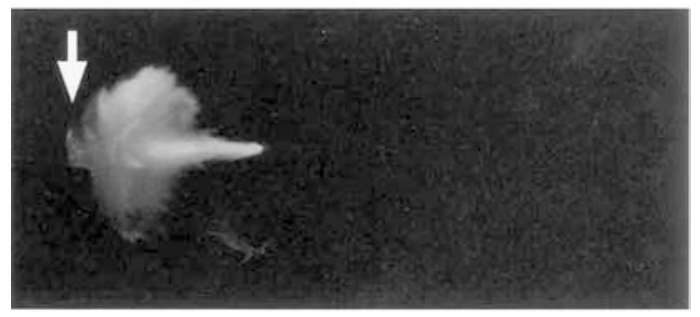

(c) Delay time $450 \mu \mathrm{s}, \mathrm{V}_{\mathrm{pro}}=180 \mathrm{~m} / \mathrm{s}$

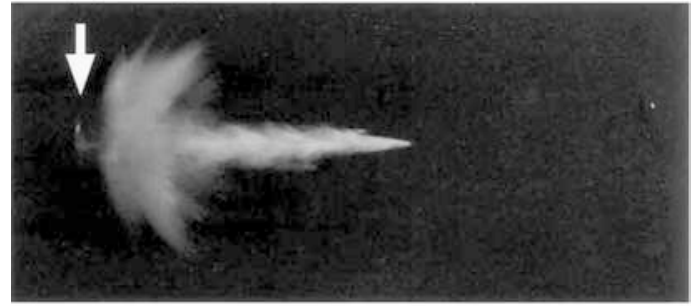

(d) Delay time $500 \mu \mathrm{s}, \mathrm{V}_{\text {pro }}=186 \mathrm{~m} / \mathrm{s}$

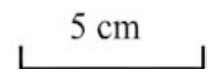

Fig. 11. Shadowgraphs of supersonic water jets from a 1-mm-diameter nozzle. IE method

reduction and quick atomization of the bifurcated jets so that a central jet emerges from the spray (Fig. 13h) and moves forward (Fig. 13i-j). The helical internal vortex shown in Fig. 11d and the wave shape of the jet boundary shown in Fig. 13j are associated with the helical instability and the Kelvin-Helmholtz instability (Andrew 1993; Shi et al. 2001).

When the CU method is applied, the jet bifurcation disappears (see Figs. 12 and 14). Because the liquid has already got initial velocity before emanating from the nozzle exit, all the fluid particles move forward. It is easy for this kind of jet to be atomized and disintegrate. It is evident from Fig. 12a-d that the expanded spray was much larger than the nozzle diameter, which is equal to the diameter of the thin liquid tail behind the spray. In contrast, the diameter of the spray generated by the $\mathrm{CU}$ method is larger than that generated by the IE method. This shows the role of the CU method in the liquid

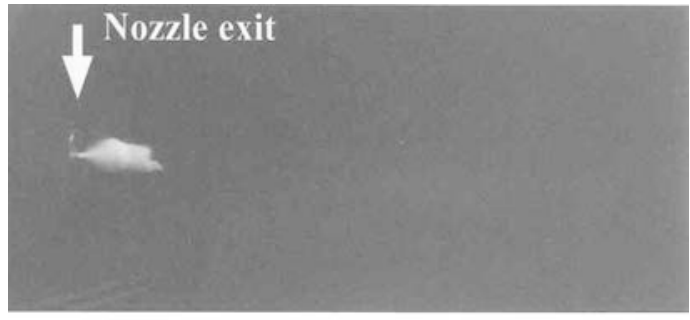

(a) Delay time $400 \mu \mathrm{s}, \mathrm{V}_{\text {pro }}=183 \mathrm{~m} / \mathrm{s}$

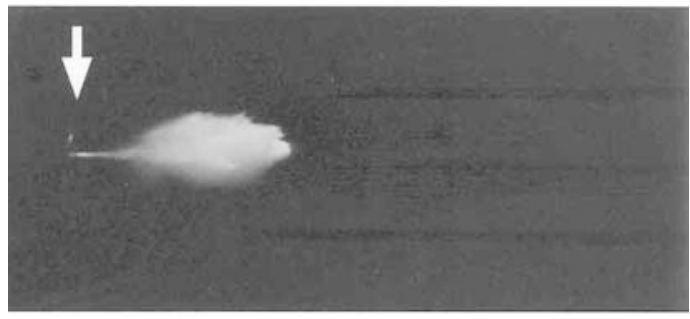

(b) Delay time $450 \mu \mathrm{s}, \mathrm{V}_{\text {pro }}=184 \mathrm{~m} / \mathrm{s}$

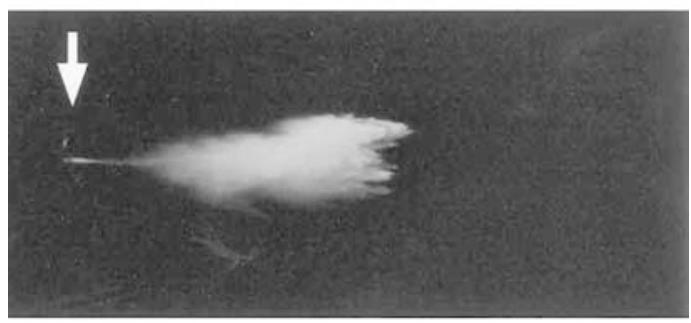

(c) Delay time $500 \mu \mathrm{s}, \mathrm{V}_{\text {pro }}=187 \mathrm{~m} / \mathrm{s}$

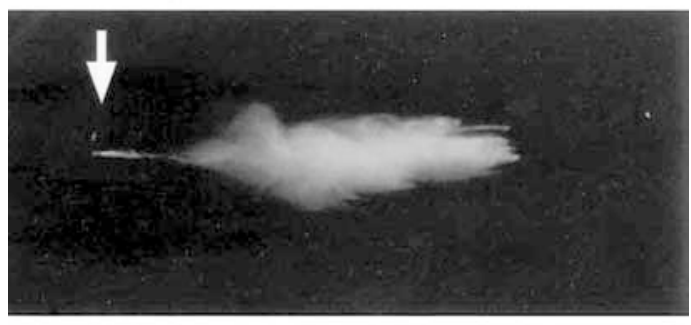

(d) Delay time $550 \mu \mathrm{s}, \mathrm{V}_{\text {pro }}=187 \mathrm{~m} / \mathrm{s}$

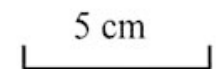

Fig. 12. Shadowgraphs of supersonic water jets from a 1-mm-diameter nozzle. CU method

disintegration and atomization. The disruption on each jet front is associated with the Rayleigh-Taylor instability (Joseph et al. 1999; Field and Lesser 1977). The schlieren photographs show the complicated shock waves system induced by the jet. In Fig. 14a, the first shock wave to appear is the transmitted shock wave which is caused by the impact on the liquid cylinder (see Fig. 3). Following the first shock wave, the second shock wave appears in Fig. $14 \mathrm{~b}-\mathrm{c}$, which is a blast wave caused by the sudden motion of the liquid in the nozzle. Then the third shock wave, which is the bow shock around the supersonic liquid jet, appears in Fig. 14d-f. Since the jet overtakes the first and second waves (Fig. $14 \mathrm{~g}-\mathrm{j}$ ), the bow shock interacts with these waves. In Fig. 14i, the arrow marks the transmitted shock wave. It is seen from the schlieren photographs that not only does the disruption of the jet head 
(a)

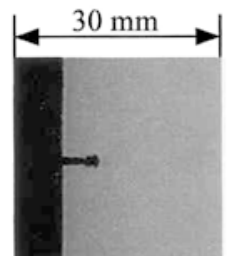

(b)

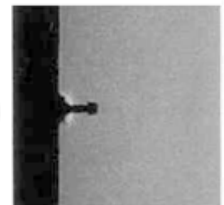

(h)

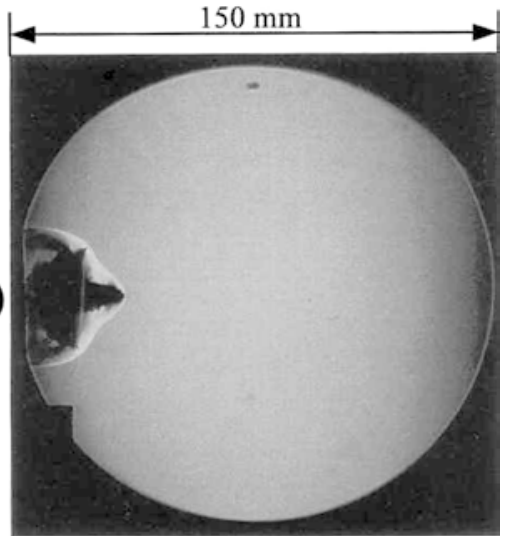

(c)

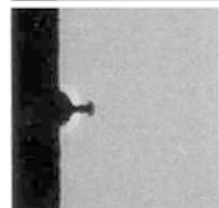

(d)

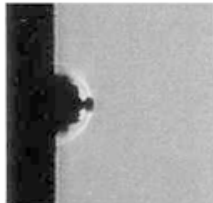

(e)

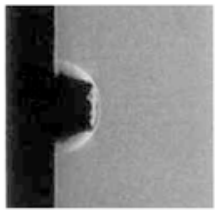

(i)

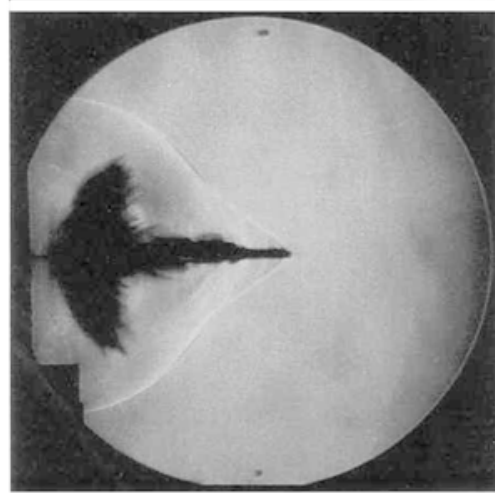

(f)

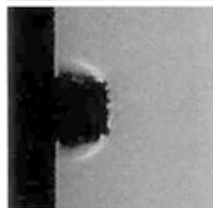

(g)

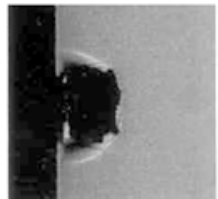

(j)

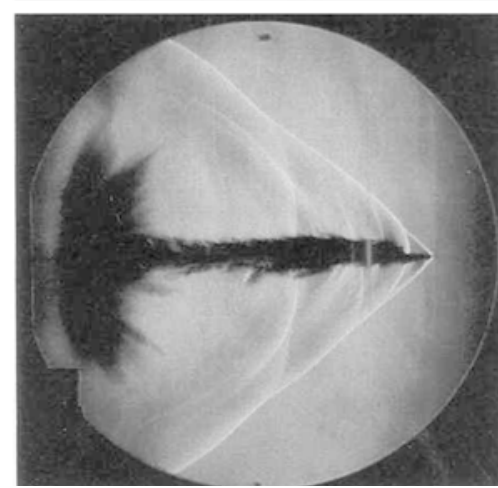

Fig. 13. Schlieren photographs of supersonic water jets from a 1-mm-diameter nozzle. IE method

cause complicated wave structure (Fig. 14i), but the wave shape of the jet boundary also causes many Mach waves (Fig. 14j). The process of a supersonic liquid jet overtaking shock waves was first stated by Shi and Takayama (1999) in their holographic observation of hypersonic liquid fuel jets. The present schlieren photography confirms the previous explanation. The aerodynamics of a supersonic projectile overtaking a gun muzzle blast wave can be seen in the work of Jiang et al. (1998).

\section{4}

\section{Conclusions}

A small high-speed liquid jet apparatus was designed to demonstrate various factors in the generation of supersonic water jets. It was found that the CU method is very effective in the disintegration and atomization of the liquid jet, but its role in jet acceleration depends on the impact momentum of the projectile, the nozzle diameter and the liquid volume. Combined shadowgraphy and schlieren (a)

(b)

(c)

(d)

(e)

(f)

(g)
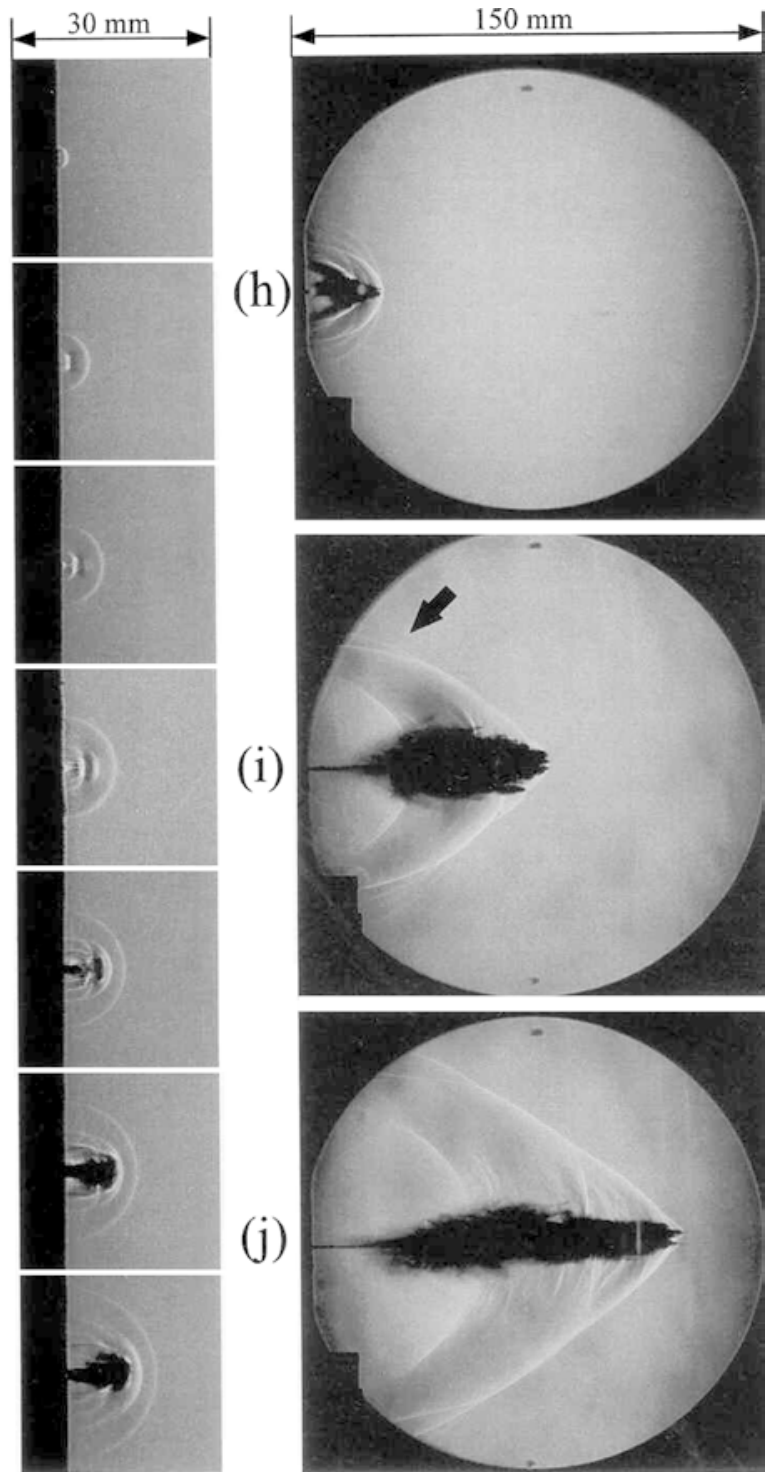

(i)

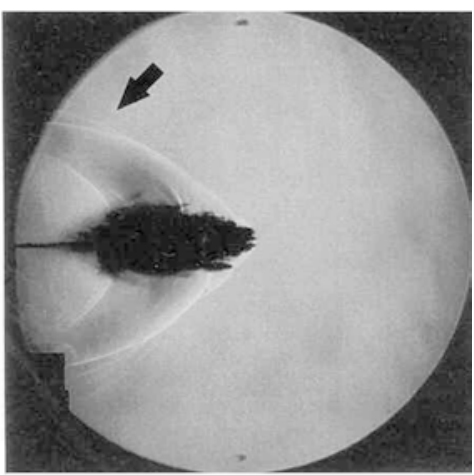

(j)

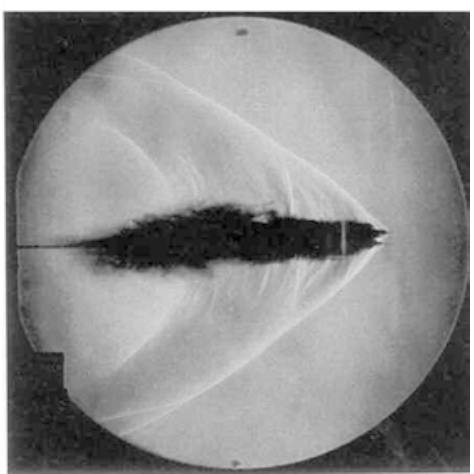

Fig. 14. Schlieren photographs of supersonic water jets from a 1-mm-diameter nozzle. CU method

photography revealed that the jet bifurcation often

accompanies the IE method, and the bifurcation is caused by the interrupted acceleration of the liquid in the nozzle as well as the discontinuity of the jet. The jet in the CU method brings about a complicated shock wave system. It is observed that the disruption of the jet head and the discontinuity of the jet can cause some new shock structures. In the relationships between the jet velocity and downstream distance, the IE method shows an advantage in keeping the jet velocity stable, since only the jet velocity of a $0.5-\mathrm{mm}$ nozzle is decreased significantly, whereas the $\mathrm{CU}$ method shows that both $0.5-$ and $1-\mathrm{mm}$ nozzles reduce the jets velocities significantly.

\section{References}

Andrew MJ (1993) The large scale fragmentation of the intact liquid core of s spray jet. Atomiz Sprays 3:29-54

Bowden FP, Brunton JH (1958) Damage to solids by liquid impact at supersonic speeds. Nature (London) 181:873

Dunne B, Cassen B (1956) Velocity discontinuity of a liquid jet. J Appl Phys 27:578-582 
Field JE, Lesser MB (1977) On the mechanics of high speed liquid jets. Proc R Soc London A 357:143

Jiang ZL, Takayama K, Skews BW (1998) Numerical study on blast flowfield induced by supersonic projectiles discharging from shock tube. Phys Fluids 10:277-288

Joseph DD, Belanger J, Beavers GS (1999) Breakup of a liquid drop suddenly exposed to a high-speed airstream. Int J Multiphase Flow 25:1263-1303

Kennedy CK, Field JE (2002) High speed liquid impact on crossed lamellar material from the shell Strombus gigas. J Mater Sci Lett 21:1457-1460

Momber AW (2001) Energy transfer during the mixing of air and solid particles into a high-speed waterjet: an impact-force study. Exp Thermal Fluid Sci 25:31-41

Pianthong K, Zakrzewski S, Behnia M, Milton BE (2002) Supersonic liquid jets: their generation and shock wave characteristics. Shock Waves 11:457-466

Shi HH, Wang TJ, Milton BE (2003a) Aerodynamic characteristics of supersonic liquid jets. Acta Aerodyn Sinica (in Chinese) in press

Shi HH, Hashiura T, Milton BE (2003b) Evaluation of the cumulative formation high-speed liquid jets. J Hydrodynam B 15(3):57-62
Shi HH, Wang XL, Itoh M, Koshiyama K, Huang Z (2001) Unsteady liquid jet flowing through a rectangular nozzle. Exp Meas Fluid Mech 15(2):59-70

Shi HH, Itoh M (1999) Generation of high-speed liquid jet from a rectangular nozzle. Trans Jpn Soc Aeron Space Sci 41:195-202

Shi HH, Takayama K (1999) Generation of hypersonic liquid fuel jets accompanying self-combustion. Shock Waves 9:327-332

Shi HH, Itoh M (1996) Design and experiment of a small high-speed liquid jet apparatus. Jpn J Appl Phys Pt 1 35:4157-4165

Shi HH, Koshiyama K, Itoh M (1996) Further study of the generation technique of high-speed liquid jets and related shock wave phenomenon using a helium gas gun. Jpn J Appl Phys Pt 135 : 4147-4156

Shi HH, Takayama K, Onodera O (1994) Supersonic diesel fuel injection through a single-hole nozzle in a compact gas gun. JSME Int J B 37:509-516

Shi HH, Takayama K, Onodera O (1993) Experimental study of pulsed high-speed liquid jet. JSME Int J B 36:620-627

Weeks BL, Klosterman J, Worsey PN (2001) Design of a hypersonic waterjet apparatus driven by high explosives. Rev Sci Instrum 72:3482-3484 